\title{
Drogen und Jugend
}

\section{Anmerkungen zum Themenschwerpunkt}

Der Zusammenhang von Drogen und Jugend ist ebenso alltäglich wie herausfordernd. Er ist einerseits alltäglich, da repräsentative Befragungen Jugendlicher stets die weite Verbreitung des Konsums illegalisierter Substanzen nachweisen. Insbesondere Cannabisprodukte und, deutlich seltener, Substanzen wie Ecstasy oder Amphetamine werden von jungen Menschen konsumiert. Dies erfolgt meist ohne größere Schädigungen oder Auffälligkeiten, in der Regel wird der Konsum kontrolliert gestaltet und von selbst wieder aufgegeben.

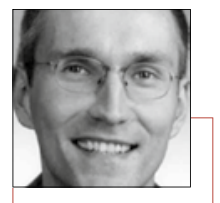

Bernd Dollinger *1973

Prof. Dr. Forschungsschwerpunkte: Theorie und Geschichte der Sozialpädagogik, Sucht- und Devianzforschung, Professionalisierung sowie Sozialpädagogik und - politik. dollinger@fb2. uni-siegen.de
Andererseits jedoch mobilisiert der Verweis auf Drogen und Jugend Szenarien der Bedrohung und der Hilfebedürftigkeit, da Jugendliche durch Abhängigkeit und Gesundheitsgefahren gefährdet zu sein scheinen. Es existieren zwar vielfältige Zwischentöne, die dieses fiktive Kontinuum von Alltag und Bedrohung ausfüllen, aber häufig zeigen sich Dramatisierungen, mit denen sich die Soziale Arbeit auseinanderzusetzen hat. Sie folgt dabei ihrerseits bestimmten Sichtweisen: In ihrem Rahmen wird häufig von Drogenkonsum als Bewältigungsverhalten, als Ausgleich individueller Defizite oder als Eskapismus, dem durch alternative Handlungschancen zu begegnen ist, ausgegangen.

Dies mag im Einzelfall richtig sein - zunächst handelt es sich jedoch um eine Perspektive neben zahlreichen anderen. Es ist genau zu reflektieren, wann und in welchen Kontexten sie angemessen ist, da es sich immerhin um die Zuschreibung handelt, dass Jugendliche den Konsum kaum selbstverantwortlich und kontrolliert handhaben könnten. Befragungen und Studien zu kontrolliertem Konsum geben Anlass zu bedenken, dass diese Annahme oftmals nicht zutrifft. Gerade die Soziale Arbeit ist dazu aufgerufen, Defizitzuschreibungen vorsichtig handzuhaben und öffentlichen Problemdiskursen durch eigenständige Reflexionen entgegen zu treten.

Dieser Schwerpunk will hierzu anregen. Obschon das Thema „Drogen und Jugend“ nicht neu ist, gibt es unverminderten Bedarf an grundlegenden Reflexionen und Positionsbestimmungen, welche die etablierte Praxis von Prävention und Drogenarbeit konstruktiv zu irritieren vermögen. Die einzelnen Beiträge unternehmen dies auf jeweils verschiedene Art und Weise:

- Bernd Werse rekonstruiert den Zusammenhang von Cannabiskonsum und Jugendkultur. Er stellt fest, dass sich aus verschiedenen historischen Wurzeln bis heute eine Art „diffuses Image“ erhalten hat, demzufolge Cannabisprodukte mit Widerständigkeit und Abgrenzung von einem ,Mainstream' assoziiert sind.

- Bernd Dollinger illustriert einen Risiko-Begriff, der von vorschnellen Problemzurechnungen Abstand nimmt. Drogenkonsum wird als Risiko beschrieben, das von Konsumenten meist

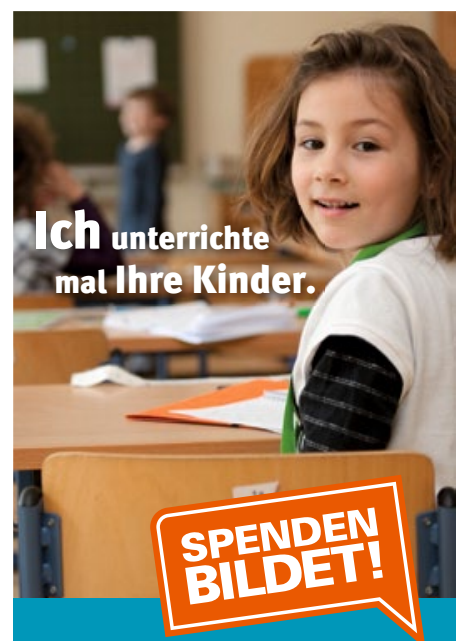

Aller Anfang ist Bildung.

Spenden Sie heute für morgen.

HypoVereinsbank

Spendenkonto 15569999

BLZ 10020890

www.spendenbildet.de

deutsche kinder- und jugendstiftung

bewusst gehandhabt wird und das nicht zuletzt durch restriktive Politikstile als Problem bestärkt werden kann.

- Gundula Barsch hinterfragt die etablierte Präventionspraxis und stellt ihr das Konzept der „Drogenmündigkeit“ gegenüber. Es versteht Drogenkonsum als subjektiv sinnvolle Handlung, die auf Kontextbedingungen angewiesen ist, die kontrollierten und bewussten Konsum möglich machen.

- Marita Benites, Geraldo Caliman und Bernd Fichtner stellen ein aktuell geplantes Projekt vor, das in Deutschland und Brasilien gegen Gewalt und Drogenkonsum vorgehen möchte. Ausgehend von einer Kritik an einer Prävention des Misstrauens und der Verbote sollen Jugendliche als eigenständige soziale Akteure ernst genommen werden. Ihnen sollen Chancen geöffnet werden, durch ästhetisch-kulturelle Praktiken Anerkennung und Selbständigkeit zu erfahren.

\footnotetext{
Abstract / Das Wichtigste in Kürze Der Drogenkonsum Jugendlicher steht zwischen Alltag und Illegalität. Die Soziale Arbeit ist diesbezüglich in einer schwierigen Lage, die sie selbstkritisch zugunsten der Perspektive Jugendlicher und ihrer Teilhabechancen auslegen sollte. .
}

Keywords / Stichworte Jugendliche, reflexive Drogenarbeit. 